\title{
El reconocimiento de las identidades educativas en la construcción del proyecto de escuela
}

\section{The Acknowledgment of the Education in the Construction of the School Project}

Cecilia Dobles Trejos ${ }^{l}$

División de Educación Básica del

Centro de Investigación y Docencia en Educación (CIDE)

Universidad Nacional

Heredia, Costa Rica

Dora María Hernández Vargas ${ }^{2}$

División de Educación Básica del

Centro de Investigación y Docencia en Educación (CIDE)

Universidad Nacional

Heredia, Costa Rica

Heidy León Arce

División de Educación Básica del

Centro de Investigación y Docencia en Educación (CIDE)

Universidad Nacional

Heredia, Costa Rica

Recibido 03 de febrero de 2009• Aceptado 17 de marzo de 2010

\footnotetext{
Antropóloga social. Profesora e investigadora de la División de Educación Básica del Centro de Investigación y Docencia en Educación (CIDE) de la Universidad Nacional, Costa Rica. Además ha sido profesora de la Escuela de Antropología y Sociología de la Universidad de Costa Rica. Se desempeña también como consultora independiente realizando investigaciones y ejecutando proyectos en los campos de la gestión cultural, derechos de poblaciones específicas y gestión de las organizaciones de la sociedad civil.

Correo Electrónico: ceciliadobles@ice.co.cr

2 Académica de la División de Educación Básica, del Centro de Investigación y Docencia en Educación (CIDE) de la Universidad Nacional, Costa Rica. Master en Pedagogía con énfasis en Diversidad en los Procesos Educativos, por la Universidad Nacional, Costa Rica, y licenciada en Ciencias de la Educación con énfasis en Educación Preescolar por la misma Universidad. Egresada del Conservatorio Castella, Costa Rica, con énfasis en Canto y Creación Literaria, continuó sus estudios de canto en la Escuela de Artes Musicales de la Universidad de Costa Rica. Autora de artículos publicados en la Revista EDUCARE sobre el tema de la diversidad. Correo electrónico: dorahv@gmail.com

3 Licenciada en Educación Especial con énfasis en Integración. Bachiller en Educación Especial. Diplomado en Ciencias de la Educación con concentración en Educación Básica I y II ciclos de la Universidad Nacional, Costa Rica. Tiene experiencia docente en instituciones costarricenses, en las que ha trabajado en educación primaria y en servicios de Educación Especial. Actualmente es académica de la División de Educación Básica del Centro de Investigación y Docencia en Educación (CIDE) de la Universidad Nacional, Costa Rica. Labora para el Ministerio de Educación Pública, Costa Rica, en el área de Retardo Mental en el servicio de Estimulación Temprana. Cursa la Maestría en Pedagogía con Énfasis en Diversidad de los Procesos Educativos. Correo electrónico: heidyleon15@hotmail.com
} 
Resumen. El presente artículo recoge las ideas centrales construidas durante un proceso de investigación que permitió una aproximación al estudio de la construcción de las identidades escolares. El abordaje que se realiza parte, no solamente de un análisis sobre la forma como se construyen y se articulan las identidades convergentes en la dinámica de una institución educativa y su relación con la propuesta educativa nacional, sino de la identificación, a partir de ese análisis, de algunas posibles líneas de acción en torno a la formación de profesionales en educación.

Palabras clave. Identidad, identidad escolar, proyecto de escuela, formación en educación, diversidad.

Abstract. This article collects the main ideas constructed during a research process that allowed an approach to the study of the construction of school identities. The approach undertaken starts off not only from an analysis of the way in which the identities that converge in the dynamics of an educational institution and their relationship with the national educational proposal are constructed and articulated, but also the identification, starting from that analysis, of some possible lines of action on the formation of education professionals.

Key words. Identity, school identity, school project, teaching training, diversity.

\section{Introducción}

La educación superior y, particularmente, la universidad pública, en tanto formadora de profesionales en diferentes disciplinas tiene una gran responsabilidad en la transformación social y cultural de nuestro país.

La realidad en nuestras comunidades, desde su diversidad cultural, plantea, cada vez con más urgencia, que se reivindiquen las particularidades culturales, o sea, los espacios a partir de los cuales los pueblos pueden reafirmar sus identidades para defenderse y mantenerse firmes ante el proceso de globalización. Se suma a esto el hecho de que, en la actualidad, las poblaciones educativas se develan como heterogéneas, en las que convergen grupos humanos que provienen de diferentes barrios, ciudades, etnias y países, y conforman un mapa pluricultural. Esta vieja situación y nueva develación no ha sido abordada de manera satisfactoria desde el sistema educativo, lo que presiona a repensar la educación desde la realidad que hoy se expresa en los ámbitos escolares.

Esta situación pone un claro reto a las universidades que preparan a los profesionales en educación: dar a los futuros educadores las herramientas necesarias para que tengan la sensibilidad y las destrezas para reconocer la diferencia, y a partir de esto, construir proyectos educativos acordes con las necesidades de las comunidades donde están las escuelas en las que van a trabajar. La formación, así concebida, debe retomar los saberes populares y científicos generados tanto en los espacios informales como en los formales y comprender, de esa manera, el hecho educativo en forma holística, en el que cada profesional incorporado a la vida comunitaria participa y es participado en su reconstrucción.

Con los objetivos, no sólo de analizar la forma como se construyen y se articulan las identidades que convergen en la dinámica de una institución educativa y su relación con la propuesta educativa nacional, sino también de identificar posibles líneas de acción en torno a la formación de profesionales en educación, se inicia nuestra ruta investigativa.

\section{La construcción de identidades escolares: una experiencia concreta}

Este artículo recoge las ideas centrales construidas durante un proceso de investigación, que permitió una aproximación al estudio de la construcción de las identidades escolares. La institución 
educativa que se escogió para llevarlo a cabo fue la Escuela Laboratorio de Heredia, pues en ese momento estaba poniendo en práctica un nuevo modelo pedagógico. Este fue un plan piloto, basado en la filosofía del lenguaje integral y con la utilización de unidades integradas, cuyo fin fue responder a las necesidades e intereses de los estudiantes de la institución. Con la puesta en marcha del modelo, se procuraba recurrir a diferentes métodos y técnicas pedagógicas que se adecuaran a las demandas de la sociedad actual.

El modelo cumple a cabalidad con la misión y la visión de la Escuela Laboratorio de Heredia, que abarca los contenidos programáticos del Ministerio de Educación Pública (MEP) y brinda a sus docentes de todas las materias, la posibilidad de trabajar con un eje común que, según Espinoza, Fuentes, Hernández y Jiménez (2004), parte del interés del estudiantado. De acuerdo con estos autores, su puesta en marcha promueve la formación integral del estudiante, y retoma y cumple con los objetivos generales para los cuales fueron creadas las escuelas laboratorio.

Sin embargo, en el momento de la investigación, el modelo estaba generando, al exterior y al interior del centro educativo, una diversidad de conflictos entre los diferentes actores, lo que evidenció las fortalezas y las debilidades con que cuentan tanto docentes como administrativos, para poner en práctica proyectos que no tienen el total apoyo del MEP.

Lo anterior se puede observar, claramente, en la discusión que planteaban los líderes del nuevo modelo pedagógico a las autoridades del MEP. La institución educativa buscaba, por un lado, el aval del MEP para poder legitimar su proyecto educativo frente a otros; pero, por otro, también, quería establecer una diferencia clara ante la propuesta tradicional del MEP, para constituirse en una institución que sirviera de ejemplo en el ámbito regional e, inclusive, en el nacional.

Asimismo, al interior del centro educativo, se suscitó otro conflicto entre los que querían romper con el modelo tradicional del Ministerio de Educación y los docentes que no querían aceptar la nueva propuesta. El enfrentamiento se configura como una pugna de poder y se convierte en un asunto eminentemente político. Es, por tanto, conveniente reconocer que las fuerzas o los pulsos internos vividos en el centro educativo podrían, eventualmente, paralizar el proyecto educativo de centro.

Internamente, se evidencia otro conflicto entre las líderes del cambio y los padres de familia, quienes sienten inseguridad ante la propuesta que se gestiona. El mayor temor de éstos se manifiesta porque se debe buscar una legitimidad del proyecto ante el MEP.

Como se ve, hay una serie de relaciones conflictivas, cuya presencia ha permitido perfilar la identidad de la institución. Esas situaciones han convertido a la escuela en un ejemplo de promoción de cambio en la región educativa de Heredia.

Es importante considerar que en toda zona geográfica de la provincia de Heredia se hace necesario reconocer a las instituciones escolares como promotoras de cambio, siempre y cuando haya una participación activa y colectiva de los docentes del centro educativo. Esos cambios deben perseguir soluciones institucionales y la gestión de nuevas propuestas, que atañen desde las teorías más elementales, los métodos, las prácticas tradicionales, las oportunidades de aprendizaje, hasta reconceptualizar y adaptar la organización de centro como un conjunto que no pueda fragmentarse en partes.

A la discusión anterior, puede agregarse lo que varios autores sostienen: el fenómeno de la discontinuidad en la relación comunidad-centro educativo-educandos. Las comunidades plantean dinámicas culturales que orientan un sentido particular, a la manera como las personas definen los procesos de socialización en los niños. En el año 2001, Cordero plantea que, actualmente, la concepción de escuela que se maneja en Costa Rica establece una discontinuidad en la socialización que llega a chocar con la práctica educativa. En este artículo es importante agregar a esta discusión que 
esos procesos de socialización son el producto de las particularidades culturales en los contextos locales, y que el estudio de estos es fundamental para entender la identidad de cada institución.

\section{Entre lo nacional y lo local: ¿se invisibiliza la diversidad?}

En nuestra sociedad costarricense, el reconocimiento de la diversidad ha sido negado en contraposición de la construcción de un Estado-nación homogéneo (al respecto se puede revisar la discusión amplia que hace Sacristán, 2001, p. 103-111). Esta posición homogeneizante evidencia una contradicción con la dinámica local y, específicamente, en los centros educativos que tienen un proyecto de escuela o que tratan de construirlo. Por esta razón, los conflictos afloran entre las propuestas provenientes de las políticas y los programas del gobierno y el proyecto de escuela en las localidades. A esto se debe agregar que, dentro de la identidad de los costarricenses, el proyecto

educativo se construye en el marco de un mito, en el cual se plantea como proyecto de vida impuesto a los costarricenses la necesidad de ser educados, como una manera de romper la ignorancia, en contraste con una serie de valores autóctonos que, por no estar legitimados por el sistema formal de educación, no tienen valor.

Al respecto, el historiador Juan Rafael Quesada, en su recorrido sobre la historia de la educación en Costa Rica, se refiere a esta visión:

Existe la idea generalizada de que en Costa Rica la educación siempre ha existido ligada a la teoría, a la práctica de la democracia y al carácter civilista de la población. En realidad, a partir de la información analizada se puede concluir, que esas afirmaciones no son ciertas, que son mitologías pretenciosamente ingeniosas, que desde finales del siglo XIX hasta hoy, han fabricado los grupos en el poder. (Quesada, 2000, p. 420)

Todo lo anterior incide en la construcción de identidades negativas, en las que el fracaso escolar es el mecanismo que nutre a un gran sector de la población. La negación de la diversidad en los procesos educativos tiene consecuencias inmediatas, las cuales se expresan, con claridad, en el ascenso en los índices de rezago, repitencia, extraedad y, por último, la deserción o la expulsión del sistema educativo.

\section{Hacia dónde debemos mirar en el estudio de la identidad escolar}

De esta correlación de fuerzas entre lo local y lo nacional, no se escapa la situación educativa en nuestro país. Por esto, la pregunta que nos planteamos es: ¿cómo deberían reflejarse las propuestas y las necesidades educativas locales en el modelo educativo nacional?, ¿cómo atiende la diversidad nuestro sistema educativo?, ¿de qué manera estos conocimientos se deben incorporar a los programas de educación en nuestras universidades?

“... la construcción de la identidad es precisamente un proceso que se lleva a cabo en contraste dialógico con los demás, una 'operación basada en el juego de las semejanzas y las diferencias', un proceso continuo de formación, transformación y conservación...” (De Lucas, 2003, p. 21). Se puede rescatar, también, para la discusión, el concepto de identidad colectiva de Berger, el cual dice que: 
Ante el choque de lo idéntico, de lo semejante, con lo diferente, con la alteridad, con lo disímil, surge un "sistema dinámico de sentimientos axiológicos y de representaciones por los cuales el acto social, individual o colectivo, orienta sus conductas, organiza sus proyectos, construye su historia, busca resolver sus contradicciones y sobreponerse a los conflictos, en función de determinaciones diversas ligadas a sus condiciones de vida, a las relaciones de poder en las cuales se halla implicado, en relaciones constantes con otros actores sociales, sin los cuales ni puede definirse ni (re)conocer(se). (Berger, citado por Montero, 1987, p. 165)

Por lo regular, en esas relaciones o interacciones de diversos actores, se presenta la confluencia de los sentidos que las personas otorgan, usualmente, a su realidad, y que desde una visión de aceptación o rechazo facilitan la articulación y la construcción de redes dinámicas que posibilitan la conformación de las identidades culturales. Esta permanente red de significaciones está ligada a las condiciones de vida de cada actor y, como ya se dijo, están dadas por procesos históricos que tienen distintas profundidades.

Desde este punto, se plantea la necesidad de reconocer cuál es el proyecto identitario que se hace evidente en la noción de escuela o comunidad de un centro educativo, y entender, en ese marco, la visión orientadora de las acciones de quienes participan vinculados a ese proyecto educativo. Es nuestro interés reafirmar, de nuevo, que para la existencia de identidad debe haber un proyecto, una visión estratégica que guíe las acciones hacia una meta. Esa meta, la mayoría de las veces, es un imaginario construido por el grupo o la persona y, por medio del cual, se representa a sí mismo $\mathrm{y}$ ante otros grupos.

En la construcción de ese proyecto, se manifiestan, claramente, los diversos actores del centro educativo, quienes toman posiciones distintas y proponen acciones que generan conflictos; pero que evidencian la posibilidad, también, de una búsqueda de metas comunes. En la articulación del proyecto que se construye a partir de cada uno de los intereses que conforman el centro, la identidad del centro educativo no es sinónimo de homogeneidad,. Se presentan algunos conflictos que evidencian la manera particular de cada persona de vivir y de definir el papel que debe tener la educación en el desarrollo local, regional o nacional.

Conocer las identidades escolares permite entender la forma en que se adecuan los programas y los proyectos educativos nacionales a cada centro, identificando la manera particular en que son apropiados y resignificados por cada uno de los actores que participa del hecho educativo en las diferentes escuelas. Estos actores, a su vez, tienen identidades particulares que, en su interacción cotidiana en el centro educativo, permiten la estructuración y la construcción de un proyecto identitario escolar.

\section{El reconocimiento de las identidades educativas en la construcción del proyecto de escuela}

En este punto, es necesario retomar los conceptos propuestos por De Lucas (2003) y Montero (1987), discutidos en el apartado anterior, a los cuales podemos añadir que la identidad escolar estaría representada, precisamente, por las relaciones y estrategias de adaptación a la interacción social.

Es comprensible que en un contexto como la escuela no hay una única identidad, sino que los diferentes grupos que la conforman establecen relaciones en su espacio cotidiano y conforman imágenes de $s u$ proyecto de escuela. Las dinámicas en la construcción, articulación y estructuración 
de ese proyecto, en relación con el modelo curricular planteado por el Ministerio de Educación Pública, es un aspecto que nos interesa analizar. Al respecto, la lectura de un currículo hegemónico nacional se contrapone con la de una propuesta educativa (materializada en el Modelo pedagógico de la Escuela Laboratorio de Heredia), la cual; sin embargo, admite múltiples lecturas y ejecuciones por parte de los actores involucrados, cada uno interpretando este proyecto escolar identitario de formas diversas.

En este mismo ámbito de análisis, se ubican, además, preguntas como: ¿de qué manera el proyecto educativo local aporta en la construcción del proyecto educativo nacional y este en el local?, ¿qué proyectos identitarios se configuran como hegemónicos y confabulan en contra de la atención a la diversidad?, ¿cómo se deberían reflejar las propuestas y necesidades educativas locales, en el modelo educativo nacional?

Es necesario aclarar que no todas las escuelas del país se sienten con la libertad y la necesidad de generar un proyecto educativo propio. Lejos de procurar que cada centro educativo tenga cierto nivel de autonomía, el Ministerio de Educación Pública considera que

Los Programas de Estudio constituyen el proyecto de lo que debe ser la labor educativa en el aula, son la perspectiva, la expectativa, es decir, lo que se espera que se construya, que se aprenda, que se desarrolle. Los Programas de Estudio son la visión del pedagogo, como los planos lo son del ingeniero o el arquitecto, Más aún, son la herramienta del pedagogo, como el bisturí lo es del cirujano. (Costa Rica. Ministerio de Educación Pública, 1996, Presentación del Plan de Estudios para el Ciclo de Transición, párrafo 8)

De esta manera, la construcción de la identidad del centro escolar no empieza con la construcción de su proyecto educativo. La singularidad y la autenticidad, como dos grandes vías de construcción de la identidad, de acuerdo con De Lucas (2003), quedan excluidas del proceso. Es solamente en virtud de un documento expreso, el Decreto de las Escuelas y Liceo Laboratorio (Decreto 7125), que se da a este tipo de instituciones la libertad para “...servir de centros experimentales para nuevos métodos y técnicas pedagógicas, así como para la realización de investigaciones en que tuvieran interés el Ministerio propiamente o las Instituciones de Educación Superior para la formación del personal docente". (Espinoza et al., 2004). Aún así, en el artículo 4 de este documento, se recuerda que:

Como establecimientos oficiales, dependerán técnicamente y administrativamente del Ministerio de Educación. Las escuelas y liceos laboratorio cumplirán con el plan de estudios y programas oficiales, sin perjuicio de las variantes que fueran necesarias introducir en razón de su condición de centros experimentales. (Espinoza et al., 2004)

De manera que, las observaciones y los análisis se basan en la construcción de la identidad de un centro educativo que tiene cierto nivel de libertad para autodeterminarse, superior al de la mayoría de los demás centros en nuestro país. A pesar de eso, alguna de las disyuntivas que al momento de la investigación salió a flote fue la forma en que la institución asume los elementos divergentes entre el modelo pedagógico propio y el plan curricular nacional.

Las autoras del modelo pedagógico (Espinoza et al., 2004) hicieron una evaluación del trabajo realizado en la escuela, el cual recogía la opinión de los padres de familia, docentes y estudiantes. Como resultado, fue posible vislumbrar que, en muchos casos, existe un antagonismo, cuando no pugna de intereses, visiones, perspectivas, necesidades y expectativas entre los grupos entrevistados. 
Si bien hay puntos de encuentro y formas comunes de enfrentar el nuevo proyecto educativo, también puede notarse la gran pluralidad de ideas existente. Como afirma De Lucas (2003), la identidad "es tensión entre el yo y el otro, entre el sujeto y el objeto (...) es constitutivamente incompleta; de aquí su plasticidad, su dinamismo, su contingencia, su impureza” (p. 21).

Notamos, en primer lugar, la necesidad de asesoría y de seguimiento de los docentes, aspecto que, según ellos, les ayudaría a empoderarse. En algunas de las entrevistas que realizamos, uno de los decentes dijo:

Es importante resaltar que la Emma Gamboa (Escuela Laboratorio de la Universidad de Costa Rica) tiene muchas diferencias con la Escuela Laboratorio (de Heredia), por ejemplo, ellos tienen aprobado su modelo por el Consejo (universitario), entonces pueden hacer frente a todo este montón de críticas, de cosas negativas que se van presentando en el camino; nosotros no, entonces muchas veces nos limitamos, aunque nosotros estamos conscientes de que hacemos lo mejor que podemos, que podemos cambiar, la podemos mejorar (entrevista grupal a docentes).

Como se ve, la construcción de la identidad es un resultado de la construcción política, un proceso de conflictos y lucha entre individuos y grupos (De Lucas, 2003). Por esta razón, los docentes estaban en la búsqueda de la legitimidad legal de su proyecto, de la colaboración de otras instancias, de la aprobación de superiores jerárquicos.

Esta búsqueda en la legalidad es un instrumento de construcción de la identidad, es la búsqueda del reconocimiento: “...el derecho impone, excluye, y hace prevalecer identidades; el derecho es guardián de la identidad por medio de su función de guardián de la diferencia." (De Lucas, 2003, p. 28). Una vez que el decreto que los docentes buscaban fuera firmado y que tuvieran la asesoría que pedían, se podían sentir autorizados para ser diferentes.

La inseguridad del cuerpo docente respondía a una incertidumbre con respecto a su posición frente a las políticas curriculares y administrativas que emanaban del Ministerio de Educación. Por ejemplo, ellos trabajaban con una metodología innovadora; sin embargo, no sabían cómo afrontar las demandas de unas pruebas nacionales escritas, que causaban altos niveles de estrés en maestros, padres de familia y estudiantes. Sin el amparo de una institución de enseñanza superior o de los mismos mecanismos jerárquicos del Ministerio de Educación, la sensación fue que la escuela, en realidad, tenía un derecho limitado a construir una identidad propia. La Escuela Laboratorio de Heredia se considera diferente de las demás escuelas, y ese es un factor clave de su identidad (en función de su diferencia); pero le ha sido muy difícil construir un proyecto integrado, con base en consensos y a partir de su legitimación como espacio para la construcción curricular.

El proyecto de la Escuela Laboratorio rompe con el principio de centralización. Como anota De Lucas (2003), “...el precio de la igualdad ha sido la uniformidad impuesta y el sacrificio de las identidades que no responden al canon nacional estatal y que han sido substituidas por la imposición de una identidad de legitimidad" (p. 16). Esta escuela está en búsqueda de una autodefinición; se encuentra en una transición entre las estructuras impuestas y su propia autonomía. La construcción de un documento, en el cual se plasme el imaginario de algunos de los actores que se hallan dirigiendo el proceso, es un testimonio de este camino emprendido. Precisamente, a causa de la falta de consenso, la integración de las aspiraciones hace posible el debate y la no coincidencia.

De acuerdo con Sacristán (2001), la construcción de la identidad subjetiva nos puede hacer conscientes de diversas dimensiones, tanto sociales como individuales, que nos constituyen. Primero, las autoras del documento Modelo pedagógico institucional, echaron un vistazo al pasado, a las 
raíces de su diferencia con otros centros educativos. De esta forma, reconstruyeron la historia de las escuelas laboratorio (bases en la tradición y la historia). La identidad está conformada por imágenes (de quiénes somos, de dónde venimos, de cómo nos vemos, etc.). Estas imágenes tienen su origen en relatos o explicaciones de por qué somos esto y no lo otro, o de por qué somos así y no de otra forma.

Luego se continuó con una búsqueda de las bases del modelo (construcción de las bases epistemológicas). Después se hicieron gestiones en el Ministerio de Educación para la aprobación del documento construido (búsqueda de la legitimidad política).

A continuación, se planteó una auto y mutua capacitación con lecturas pertinentes a los contenidos del modelo (continúa la búsqueda de claridad epistemológica y metodológica). Después, con la participación del grupo de docentes, se realizó una evaluación del trabajo realizado con las unidades integradas (búsqueda de consensos).

Se continuó con gestiones para la firma de un Convenio de Cooperación entre la Universidad Nacional y la Escuela Laboratorio de Heredia, con el fin de mantener la cualidad de laboratorio pedagógico y la calidad académica (afirmación de su identidad frente a las fuerzas homogeneizadoras). Posteriormente, se prosiguió con una evaluación (a la que nos hemos referido antes), con la participación de los sujetos involucrados en el proceso educativo, a saber: estudiantes, docentes y padres de familia (reflexión sobre lo construido).

Gran parte del esfuerzo por construir el modelo pedagógico de la escuela laboratorio nace frente a la amenaza de no ser contemplados como una escuela diferente por parte del Ministerio de Educación, a causa de la ausencia de un proyecto distintivo. Entonces, empieza un proceso de construcción.

De acuerdo con lo que afirmaban los mismos docentes en las entrevistas realizadas, la Escuela Laboratorio, luego de la separación que tuvo de la Universidad Nacional, fue perdiendo su visión original. Esto pudo haber sucedido, en gran parte, porque las estructuras y las dinámicas, desde el nombramiento de directores y docentes hasta las exigencias curriculares, eran las mismas que para una escuela común. De esta forma, es posible ver cómo un currículo hegemónico invisibiliza las diversidades, coarta la autonomía y desfigura la singularidad. Existen muchas barreras administrativas y curriculares para la elaboración de un proyecto de centro que contribuya en la construcción de las identidades escolares. Sin embargo, de acuerdo con Fullan y Benett, citados por Pérez (2000), en el marco de la discusión sobre las reformas y los cambios educativos:

(...) desde la perspectiva educativa los cambios y reformas deben pretender no la ejecución minuciosa y fiel del programa previsto por la Administración, sino el desarrollo de las capacidades individuales y colectivas y la facilitación de las condiciones estructurales y organizativas para que la institución escolar pueda decidir, de manera autónoma y competente y sin perder de vista sus finalidades educativas, las transformaciones que requiere la evaluación del contexto. El cambio educativo no se reduce a la elaboración de nuevas estrategias y procedimientos para ejecutar reformas impuestas desde fuera, sino que tiene que ver con el desarrollo por parte de los individuos implicados de estrategias personales para responder a, e influir sobre, el impacto de los cambios estructurales y culturales del escenario escolar y social. (p. 144).

Surge, además, la necesidad de replantearse la importancia que tiene la construcción de estas identidades escolares como fundamento para la construcción de identidades sociales o políticas de mayor impacto. El organigrama muy vertical de la Escuela Laboratorio (Modelo pedagógico) nos hace ver que las estructuras necesitan moverse, conscientemente, de forma más interactiva, para permitir una vivencia más democrática. 
Podríamos establecer el nivel de descentralización con que cuenta la Escuela Laboratorio, de acuerdo con las características de una escuela centralizada, según Moschen (1997):

- Instituciones escolares que se comportan como simples "receptoras de órdenes superiores" (último eslabón de una cadena).

- Modelo de organización escolar estereotipado que se aplica uniformemente en cualquier lugar geográfico y contexto social.

- Instituciones que no investigan las necesidades reales de los destinatarios del servicio.

- Fuertes estructuras de supervisión ajenas a ellas mismas.

- Instituciones con escaso sentido de competencia, carentes de objetivos de rendimiento, incapaces de autoevaluarse y medirse con otras instituciones de su especialidad o de su zona de influencia.

- Instituciones cuyo personal no es seleccionado con la participación de los responsables de la conducción escolar. (p. 7)

También podemos conocer el nivel de descentralización que ha logrado la Escuela Laboratorio, si tomamos en cuenta las características de una escuela descentralizada:

- Capacidad, por parte de la institución, para interpretar su misión específica dentro de un determinado tiempo y lugar.

- Capacidad para elaborar y seleccionar las mejores estrategias frente a los problemas educativos propios y específicos.

- Capacidad para incorporar en su proyecto educativo y "mensaje curricular" objetivos y acciones acordes con las estrategias propias y específicas.

- Capacidad para diseñar sus estructuras funcionales y gestionar su propio proyecto.

- Capacidad para establecer criterios de calidad y evaluar "objetivos de rendimiento".

- Capacidad para seleccionar al personal y evaluar su desempeño de acuerdo con las características del servicio que se quiere prestar. (Moschen, p. 9)

En opinión de este autor, algunas de las acciones previstas deben iniciarse en un equipo especial de trabajo, al cual se denominará equipo promotor; otras acciones se llevarán a cabo en instancias participativas más amplias. La función del equipo promotor (en el caso de la Escuela Laboratorio: el Comité de Mejoramiento Profesional) no es monopolizar la transformación institucional ni mucho menos la de suprimir o manipular la participación. Su presencia responde a la necesidad de crear un cuerpo institucional, en el que estén representados todos los grupos y, también, gestionar diversos aspectos que se relacionan con el proceso global de la transformación y la construcción de esa identidad.

La necesidad de autonomía de los centros educativos es cada día mayor. En el caso concreto de la Escuela Laboratorio, esta necesidad se refleja en que la institución acoge niños y niñas de casi todos los cantones de Heredia, por lo que, se supone, la diversidad ha de ser mayor, en tanto más comunidades se encuentren representadas. Sin embargo, nuestra política educativa no cuenta con un sistema estructural de apoyo que haga posible la existencia de comunidades educativas descentralizadas y autónomas.

La conformación heterogénea de las comunidades educativas se ve opacada frente al Estadonación homogéneo. Como afirma Villoro (1998): 
El Estado-nación es concebido como una asociación de individuos que se unen libremente por contrato; en este sentido, la sociedad no es vista ya como la compleja red de grupos disímbolos, asociaciones, culturas diversas, estamentos que se han ido desarrollando a lo largo de la historia, sino como una suma de individuos que acuerdan hacer suya una voluntad general. Solamente así se pasaría de una asociación impuesta por una necesidad histórica a una asociación basada en la libertad de los asociados. (p. 1)

Esto genera, en este caso específico, un conflicto entre las propuestas que derivan de las políticas y de los programas educativos y el proyecto de escuela local. De forma que, por ejemplo, la propuesta metodológica particular choca con la propuesta estandarizada de evaluación. Por esta razón, hay pocas posibilidades para que el proyecto educativo local ayude en la construcción del proyecto educativo nacional. El proyecto identitario del Estado se configura como hegemónico y atropella la construcción de identidades escolares, al menos, de aquellas con una base epistemológica, metodológica o curricular divergente.

Si en lo social y en lo cultural camináramos hacia la construcción de una sociedad democráticamente plural, estaríamos previendo que la educación formal pueda ofrecer su aporte en la construcción de estas dimensiones de la identidad. Si los centros educativos no cuentan con ciertos niveles de autonomía y descentralización, si se mantiene la desconfianza en las competencias profesionales del docente (y, en este caso, es posible observar cómo esa identidad docente se encuentra mutilada de muchas maneras) y si hay resistencia, en los cargos directivos y administrativos del sistema, por delegar el control en instancias sociales, será casi imposible el tratamiento educativo del cambio en las escuelas y en el sistema educativo y, en definitiva, en el sistema social (Pérez, 2000).

Frente a estas reflexiones, Goodman (citado por Pérez, 2000) considera que:

La educación de los niños/as es un complejo fenómeno que implica estructuras organizativas, identidades personales, dinámicas interpersonales y comunicaciones simbólicas. Comprender lo que sucede en una determinada escuela no es fácilmente accesible por medios simples y directos. En consecuencia, la educación como experiencia viva debe comprenderse a través de la observación de las personas, cuando se implican en diferentes tipos de experiencias comunicativas, cuando manifiestan sus identidades personales, cuando crean estructuras, rituales y símbolos que expresan sus valores e ideas. (p. 147)

\section{A modo de conclusión}

En uno de los apartados, nos preguntábamos de qué manera esta perspectiva sobre las identidades educativas en la construcción del proyecto de escuela pueden ser incorporadas dentro de los programas de educación de nuestras universidades. Para este cuestionamiento no existe una respuesta fácil ni única. Pero, a partir del proceso de investigación, fue posible identificar algunas formas de solución.

En primer lugar, deberíamos considerar la investigación como una actividad clave para el desarrollo de un pensamiento pedagógico, que sea capaz de inquirir en los fenómenos sociales, en los cuales se hallan inmersos los procesos educativos, desde un enfoque dialéctico. Esto orienta a lo que podría constituirse en una segunda línea de formación: la consideración del currículo como un campo político y de los docentes como sujetos políticos. En este sentido, los docentes se constituyen en un eslabón importante en el proceso de construcción de identidades escolares, cuando, dentro de una comunidad educativa, intervienen en el diseño curricular, a partir de las aspiraciones y la 
constitución de esa comunidad educativa. La escuela no se considera, así, como institución inerte, ahistórica, inmóvil, estática, sino como espacio dinámico de intercambios en la construcción de la sociedad (local y nacional). De esta manera, abordamos la que sería la tercera línea propuesta para la formación de docentes: la necesidad de formar docentes que sean capaces y que gusten de trabajar en redes pedagógicas.

La construcción de proyectos educativos locales requiere de la capacidad de interacción, de negociación, de diálogo, de debate. Resulta, en este sentido clave, la capacidad del docente (en trabajo conjunto con los directores de las instituciones) para participar en la articulación y cohesión de intereses, esfuerzos y búsquedas comunes; que les permita robustecerse como sujetos y como sujetos colectivos.

Una escuela con rostro, con identidad, requiere docentes empoderados; docentes que asuman la escuela, no como un lugar aislado de la vida social y del devenir histórico, sino como un espacio de construcciones colectivas. Pese a la existencia de un proyecto educativo nacional, que bien puede ser deseable, los centros educativos requieren de espacios para la reivindicación de sus identidades locales. Un docente capaz de integrarse en la construcción de proyectos educativos de centro, de forma dialógica y propositiva, puede contribuir en mucho en la visibilización de nuestro pluralismo.

\section{Referencias bibliográficas}

Cordero, T. (2001). Desencuentros entre prácticas escolares y vivencias familiares. En Actualidades investigativas en educación. Vol. 2, N 2, Año 2001. IIMEC, UCR. Versión electrónica: http:// revista.inie.ucr.ac.cr/articulos/2-2002/archivos/desencuentros.pdf. Consultada enero 2007.

Costa Rica. Ministerio de Educación Pública. (1996). Programa de Estudios. Ciclo de Transición, Educación Preescolar. San José: El Ministerio.

De Lucas, J. (2003) Globalización e identidades. Barcelona, España: Editorial Icaria-Antrazyt.

Espinoza, S., Fuentes, I., Hernández, M. \& Jiménez, G. (2004). Modelo pedagógico institucional. Documento no publicado.

Montero, M. (1987). Psicología política latinoamericana. Caracas Venezuela: Editorial Panapo.

Moschen, J. C. (1997) La escuela con proyecto propio. (2 $2^{\mathrm{a}}$ ed.) Buenos Aires, Argentina: El Ateneo.

Pérez, Á. (2000) La cultura escolar en la sociedad neoliberal. Madrid, España: Editorial Morata.

Quesada, Juan Rafael (2000) La educación en Costa Rica: 1821-1914. En: Costa Rica, Estado, economía, sociedad y cultura: desde las sociedades autóctonas hasta 1914. Botey, Ana María, Coordinadora. Ed. Universidad de Costa Rica.

Sacristán, J. G. (2001). Educar y convivir en la cultura global. Madrid, España: Editorial Morata.

Villoro, L. (1998). Del Estado homogéneo al Estado plural. En: Estado plural, pluralidad de culturas (pp. 13-62). México: Paidós / UNAM. 